\title{
Design of textured surfaces for super-hydrophobicity
}

\author{
PRITHVI RAJ JELIA, AMIT AGRAWAL, RAMESH K SINGH and SUHAS S JOSHI*
}

Department of Mechanical Engineering, Indian Institute of Technology Bombay, Powai, Mumbai 400076, India

e-mail: ssjoshi@iitb.ac.in

MS received 16 January 2016; revised 4 January 2017; accepted 30 April 2017; published online 11 November 2017

\begin{abstract}
Although the Cassie-Baxter and Wenzel equations predict contact angles for relative dimensions of micro-pillars on textured surfaces, the absolute pillar dimensions are determined by trial and error. Alternatively, geometries of natural super-hydrophobic surfaces are often imitated to design textured surfaces. Knowing the limitations of both the approaches, this work presents a constraint minimization model on the basis of CassieBaxter equation to determine the absolute dimensions of square micro-pillars on a textured surface so as to maximize the contact angle. The constraints are derived based on the limiting physical conditions at which spontaneous breakdown of super-hydrophobicity takes place. The single-droplet numerical simulations on textured surface gave the duration for which super-hydrophobicity is sustained. The model demonstrated that the round edged pillars, arising out of fabrication imperfections, reduce the height of the pillars without significantly compromising on the contact angle. The measurement of contact angle on the fabricated textured surfaces was found to be in agreement with the model predictions when the fabricated pillars had fairly uniform dimensions. The proposed approach is sufficiently general that its application can be extended to design other textured surfaces.
\end{abstract}

Keywords. Textured surfaces; super-hydrophobicity; contact angle; Cassie-Baxter state; Wenzel state; singledroplet simulation.

\section{Introduction}

Super-hydrophobic surfaces are defined as the surfaces that exhibit a contact angle greater than $150^{\circ}$ with water droplet resting on them. They also exhibit very low contact angle hysteresis, causing the water droplet to fall off quickly. Super-hydrophobic surfaces have many practical applications such as enhanced water proofing for fabrics, anticontamination, lab-on-chip gene delivery systems [1], selfcleaning window panes, windshields and mirrors, corrosion-resistant surfaces [2], plastic optics with high light transmission [3], anti-fog material for eye glasses and swimming goggles [4] and other applications such as in solar systems. The present work is motivated by the property of super-hydrophobic surfaces in reducing shear stresses at fluid-wall interfaces required in medicinal applications.

In nature, super-hydrophobicity is encountered on a number of plants and insects, such as the leaves of lotus, eucalyptus, legs of water striders and some spiders. The example of lotus leaf is often used and hence the name 'lotus effect' has become synonymous with the phenomenon of super-hydrophobicity. A close examination of these surfaces reveals the presence of micron-sized

*For correspondence structures, which sometimes are covered with a second layer of nano-sized structures. The features are further coated with epicuticular wax film rendering super-hydrophobicity to the surfaces [5]. Researchers have tried to fabricate these features, micro-structures in particular, to achieve artificial super-hydrophobic surfaces.

A number of methods are used to fabricate such textured surfaces. Super-hydrophobic film was prepared by chemically adsorbing myristic acid (n-tetradecanoic) onto a copper wafer [6]. Flexible and transparent super-hydrophobic surfaces can be fabricated by imprinting and anchoring of conformally uniform poly(dimethylsiloxane) [3]. Huang et al [4] fabricated a surface with micro-bowl arrays using three-dimensional diffuser lithography using randomized UV light. A mixture of two colloidal suspensions of Teflon and larger sacrificial polystyrene beads gives a large contact angle of $\sim 170^{\circ}$ [7]. Super-hydrophobic surfaces fabricated by chemical methods do not remain intact for long duration of time and their fabrication is limited to relatively small surface areas. Tang et al [8] fabricated micro-patterns on stainless steel by focusing high-energy femto-second laser beam. A fluorinating agent was used to fabricate super-hydrophobic surfaces on copper and aluminium [9]. Another method to achieve super-hydrophobicity is to generate densely packed aligned carbon nano-tubes (ACNTs) over a low free energy material such 
as silicon wafer [1, 10, 11]. Yoon et al [12] used a modified electrostatic process to achieve super-hydrophobicity on poly ( $\varepsilon$-caprolactone).

The explanation for the increase in the contact angle or hydrophobicity on the textured surfaces is usually offered by two classical theoretical models, the Cassie-Baxter model and the Wenzel model. In the Wenzel model, water completely penetrates the surface cavities. On the other hand, in the case of Cassie-Baxter model, water touches only the top surface of the micro-pillars, thereby leaving air to remain trapped within the surface cavities. It is noted that water droplets on super-hydrophobic surfaces that exhibit large contact angles are in the Cassie-Baxter state. Therefore, the trapped air in the surface cavities is attributed to be the primary reason for super-hydrophobicity of these surfaces.

Although super-hydrophobicity has been achieved on various surfaces, there are a number of issues, such as the following: (i) generating super-hydrophobicity over large surfaces is rather difficult, (ii) modelling super-hydrophobicity of dynamic fluid flow has not been undertaken and (iii) fabrication of a surface that retains super-hydrophobicity over long duration remains a challenge. In the last case, the less stable Cassie-Baxter state is replaced by the more stable Wenzel state, leading to the phenomenon called 'spontaneous breakdown' of super-hydrophobicity. Various theories have been proposed to explain this spontaneous breakdown [13, 14]. Simulations have been conducted $[15,16]$ for single-droplets to study these effects. Klein et al [13] reasoned that breakdown of super-hydrophobicity occurs when the micro-pillars are separated by large distances. In such cases, the upward surface tension forces can no longer sustain the weight of the liquid droplet above the micro-pillars. The Cassie-Baxter equation $\cos \theta^{*}=-1+$ $\phi(1+\cos \theta)$ predicts that the apparent contact angle $\theta^{*}$ increases monotonically as the pillar spacing ratio $(\phi)$ decreases, where, $\theta$ is the contact angle on a flat surface; $\phi$ is a ratio of apparent projected area in contact with the liquid droplet to the total area. It is a function of the pillar width $a$ and spacing between the pillars $b$ (see figure 1). In fact, $\phi$ is the only governing factor that controls the contact

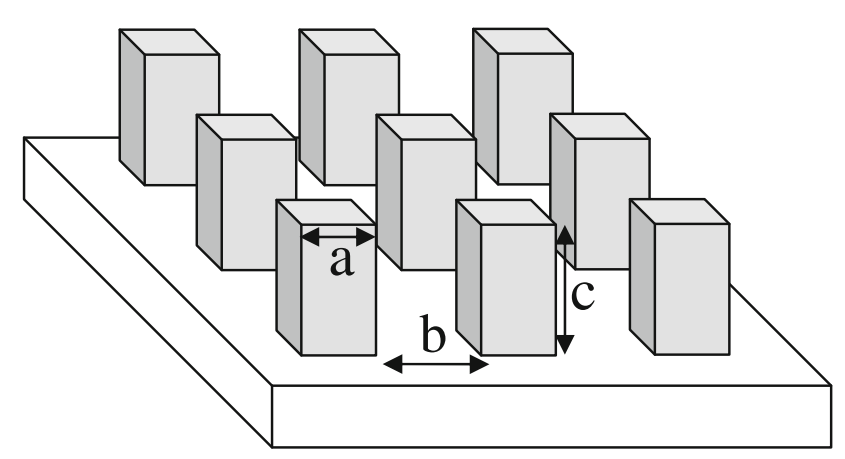

Figure 1. Model showing microscopic square pillars arranged in square array. angle for a liquid-solid pair. Below a certain critical spacing ratio, the contact angle cannot be increased; breakdown of super-hydrophobicity occurs beyond this critical angle [13]. These ideas have been tested using numerical simulations in the present work.

Although the Cassie-Baxter equation predicts the contact angles for relative values of pillar dimensions $a$ and $b$, the absolute values of $a, b$ and $c$ (figure 1) are mostly found out by trial and error method. Alternatively, the dimensions of the naturally occurring super-hydrophobic surfaces have been imitated while designing textured surfaces. Both these methods have severe limitations. The primary objective of this work is to determine the absolute pillar dimensions $a$, $b$ and $c$ by treating it as an optimization problem. The dimensions have been arrived at by maximizing the contact angle made by a single droplet with the textured surface. At the same time, the proposed approach considers the superhydrophobic state to be stable. This is achieved by analytically modelling the wetting phenomenon by a singledroplet resting over a surface textured with an array of square pillars. A constraint minimization problem based on the Cassie-Baxter equation is formulated with the objective of maximizing the contact angle made by the droplet over the textured surface. The constraints are derived based on limiting physical conditions at which spontaneous breakdown of super-hydrophobicity takes place. The model also considers the effect of manufacturing limitations, such as rounding of pillar corners (instead of sharp corners), on the contact angle. The optimum dimensions are again obtained using the analytical model. Surfaces fabricated with the optimum dimensions are experimentally tested for superhydrophobicity and the results are validated against the predictions from the model. Our approach is therefore believed to be sufficiently general, in the sense that cases other than those tested here can be examined using the same optimization approach.

The outline of the paper is as follows. Analytical solutions for the case of sharp and round edged pillars are developed in section 2 . The constraint minimization problem is formulated and solved in section 3. Section 4 presents the simulation of a single droplet falling over the textured surface using a numerical solver. Section 5 presents details of fabrication of textured surfaces and corresponding measurements of contact angle. The experimental and theoretical results are also compared in this section. The paper concludes with remarks in section 6 .

\section{Analytical model}

The naturally occurring super-hydrophobic surfaces have micrometre-sized textures on them. To simulate the behaviour of a droplet on super-hydrophobic surfaces, an analytical model is proposed in which a single droplet is assumed to rest on a surface bearing an array of square pillars. The objective of the model is to optimize the 


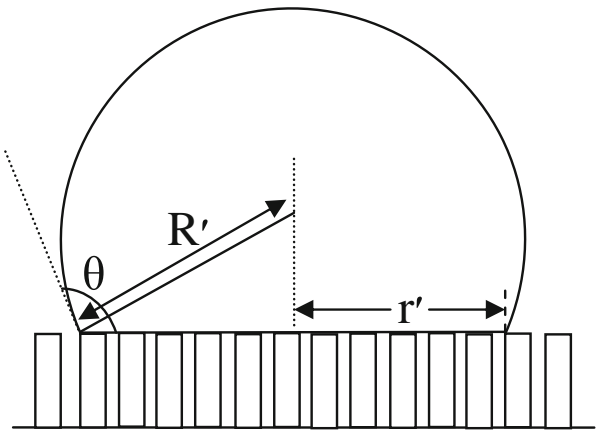

Figure 2. Droplet deposited over textured surface in Cassie state. A single droplet touches a number of pillar tops.

dimensions of the pillared structure such that the contact angle between the deposited droplet and the pillared surface is maximized.

The geometry of the surface considered in the model is shown in figure 1, where the width and height of the regularly arranged pillars are $a$ and $c$, respectively, while the spacing between the pillars is $b$. A single droplet with radius $R$ in air is deposited over the pillars (see figure 2). A constrained optimization problem is then framed to maximize the contact angle, where the objective function is based on the Cassie-Baxter equation, and the constraining inequalities are derived based on physical and thermal equilibrium conditions to ensure stability of the deposited droplet and prevent breakdown of super-hydrophobicity. The optimum pillar dimensions $(a, b, c)$ are obtained for this maximum contact angle. The optimization problem is then solved using the 'trust region reflective' algorithm using the Mat${ } \mathrm{lab}^{\circledR}$ software package. Further, manufacturing considerations are taken into account in the model by considering round edged pillars and by following a similar procedure described earlier to determine the maximum contact angle and the optimum pillar dimensions for this case.

\subsection{Assumptions}

The model is based on the following assumptions:

- The droplet is in ideal Cassie-Baxter state, i.e., the liquid does not wet the lateral sides of the pillars, but wets only the top surface of the pillars.

- The curvature of the liquid meniscus between the pillars is convex.

- The air trapped between the liquid meniscus and solid substrate is at the atmospheric pressure (initial pressure). Conversely, when the droplet is deposited on the pillared surface, while it is stabilizing in the CassieBaxter state, some air escapes out, thereby allowing the liquid to overhang.

- The apparent (projected) contact area of the droplet when deposited on the textured surface is circular in shape; it implies that the contact line is circular.
- The weight of the droplet is uniformly distributed over the apparent contact area.

- The volume of the water penetrating between the pillars is negligible as compared with the total volume of the droplet.

- The curvature of the droplet deposited on the textured surface is hemi-spherical.

- The droplet size is on the order of millimetres, while the size of the pillars is on the order of micrometres. Therefore, the droplet deposited over the textured surface rests over a number of pillars.

\subsection{Derivation of inequality conditions}

The constrained minimization problem involves three inequalities derived based on the following three physical conditions:

- The lower meniscus of the droplet just touches the substrate.

- The weight of the droplet between the pillars is balanced by the upward surface tension forces.

- The dimensions of the pillared textures are such that the Cassie-Baxter state is energetically more favourable than the corresponding Wenzel state.

2.2a Lower meniscus of droplet just touches the substrate: If the lower meniscus of the droplet deposited on the textured surface touches the substrate, the Cassie-Baxter state would be replaced by the Wenzel state, causing a sudden breakdown in super-hydrophobicity [6]. Figure 3 shows a portion of the complete liquid-solid interface between four pillars (figure 4).

To prevent the lower meniscus from touching the substrate, it is required that $s<c$. Based on the geometry in figure 3 , we obtain

$$
\frac{c}{b}>\left(\frac{1-\sin \theta}{-2 \cos \theta}\right)
$$

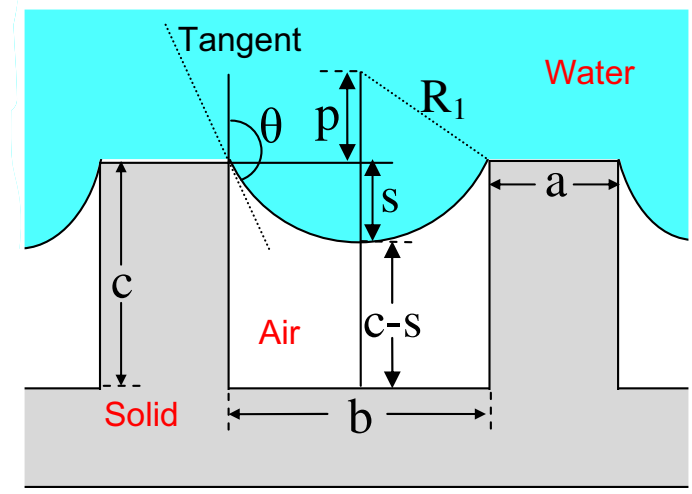

Figure 3. Schematic showing the portion of a droplet deposited on textured surface between adjacent pillars. 
Note that $\theta$ has been defined with respect to the vertical surface, since the movement of the meniscus is along the vertical wall as the droplet starts to move down into the cavity.

$2.2 \mathrm{~b}$ Droplet weight balancing: The weight of the portion of the total droplet liquid lying above each cavity is assumed to be balanced by the surface tension forces arising out of the contact made by the droplet with the unit cell shown in figure 4 . When the surface tension force exceeds the weight of the liquid above it, there is a sudden decrease in the contact angle, as the Cassie-Baxter state is now replaced by the Wenzel state; consequently, breakdown of super-hydrophobicity takes place.

The apparent contact area between the deposited droplet and the substrate is evaluated for the droplet in equilibrium, considering Cassie-Baxter state; see the schematic in figure 5. In the figure, $r^{\prime}$ represents radius of the apparent circular contact area between the droplet and the surface.

Let $R$ be the radius of spherical droplet in air, and let $R^{\prime}$ be the radius of curvature of an incomplete deposited droplet (see figure 5). From the geometry, volume of the droplet is given as follows:

$$
V=\frac{\pi}{3}\left(R^{\prime}+R^{\prime} \cos \theta^{*}\right)^{2}\left\{3 R^{\prime}-\left(R^{\prime}+R^{\prime} \cos \theta^{*}\right)\right\} .
$$

Equating this with the original volume of the spherical droplet and rearranging gives

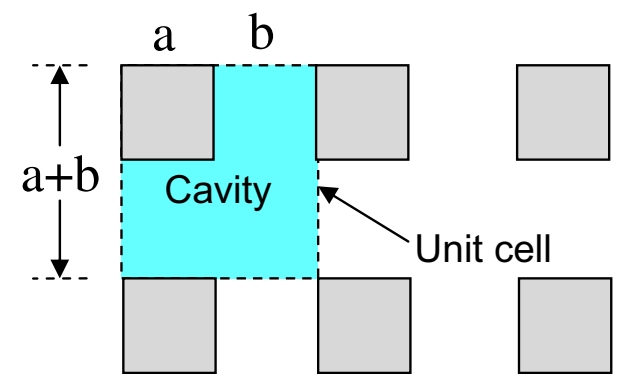

Figure 4. Schematic showing top view of pillared surface; weight balancing is done for a portion of liquid lying above a unit cell shown by dotted square.

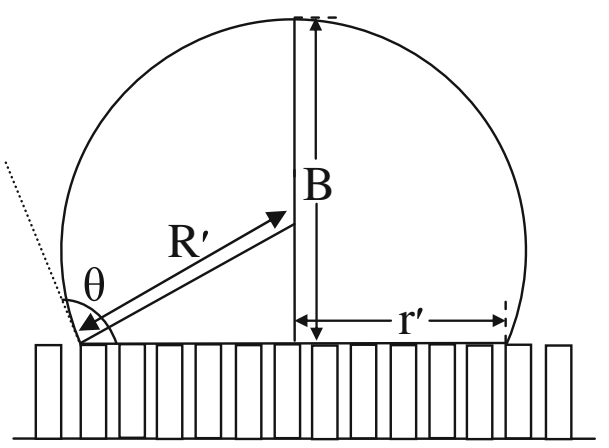

Figure 5. Schematic showing the deposited droplet having spherical curvature of a different radius $R^{\prime}$.

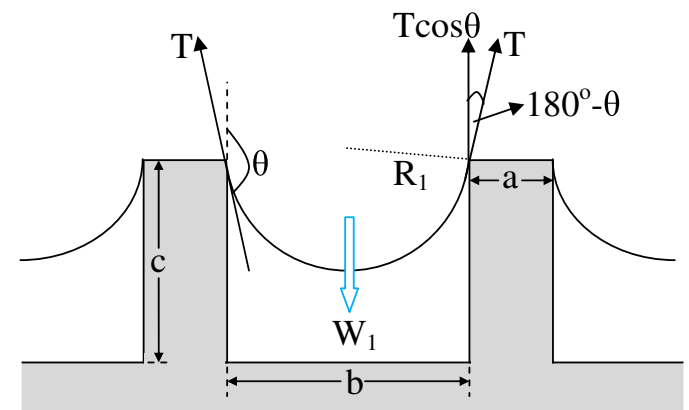

Figure 6. Schematic showing the vertical component of the surface tension force acting on the portion of the droplet present above a 'cavity'.

$$
R^{\prime}=R\left(\frac{4}{\left(1-\cos \theta^{*}\right)^{2}\left(2+\cos \theta^{*}\right)}\right)^{\frac{1}{3}} .
$$

As $r^{\prime}=R \sin \theta$, the apparent contact area between the deposited droplet and the pillared surface, given as $A=\pi r^{\prime 2}$, is found to be

$$
A=\pi R^{2} \sin ^{2} \theta^{*}\left(\frac{4}{\left(1-\cos \theta^{*}\right)^{2}\left(2+\cos \theta^{*}\right)}\right)^{\frac{2}{3}} .
$$

If the weight of the droplet $W$ is uniformly distributed over the entire contact area $A$, the weight on a cavity area $A_{1}$ (which is equal to $(a+b)^{2}-a^{2}$ ) equals $W_{1}=\frac{W}{A} A_{1}$, and is given as follows:

$$
W_{1}=\frac{\rho\left(\frac{4}{3} \pi R^{3}\right) g}{\pi R^{2} \sin ^{2} \theta^{*}\left(\frac{4}{\left(1-\cos \theta^{*}\right)^{2}\left(2+\cos \theta^{*}\right)}\right)^{\frac{2}{3}}}\left\{(a+b)^{2}-a^{2}\right\} .
$$

As the total liquid-solid contact length in one 'unit cell' is equal to $4 a$, the component of the surface tension force (see figure 6) in the upward vertical direction is given as $F_{1}=|4 a T \cos \theta|$. This force should be large enough to bear the weight of droplet over the unit cell, i.e., $F_{1} \geq W_{1}$. On substituting $W_{1}$ from Eq. (5), and rearranging, we obtain

$$
b<\left(\sqrt{\frac{-3 a T \sin ^{2} \theta^{*} \cos \theta}{\rho R g}\left(\frac{4}{\left(1-\cos \theta^{*}\right)^{2}\left(2+\cos \theta^{*}\right)}\right)^{\frac{2}{3}}+a^{2}}\right)-a .
$$

2.2c Cassie-Baxter state is more stable than Wenzel state: The surface energy associated with the Cassie-Baxter state should be lower than the energy associated with the Wenzel state, so that the Cassie state is thermodynamically more stable. Considering the surface energy of one 'unit cell', the respective surface energies (defined as the summation of the products of the interfacial surface tension and interfacial areas of all interfaces lying within one 'unit cell') can be written as 


$$
E_{w 1}=\gamma_{s l}\left[(a+b)^{2}+4 a c\right]
$$

and

$$
E_{c 1}=\gamma_{s v}\left[(a+b)^{2}+4 a c-a^{2}\right]+\gamma_{s l} a^{2}+\gamma\left[(a+b)^{2}-a^{2}\right]
$$

Here, it is assumed that the actual liquid-vapour interface area is equal to the apparent projected area. Substituting Eqs. (7) and (8) into $E_{c 1}<E_{w 1}$, and using Young's relation, $\cos \theta=\frac{\gamma_{s v}-\gamma_{s l}}{\gamma}$ [5], we obtain

$$
\cos \theta+\left(\frac{1}{1+\frac{4 a c}{b^{2}+2 a b}}\right)<0 .
$$

Equations (1), (6) and (9) give only the relative bounds on the dimensions $a, b$ and $c$. In order to obtain their values, an objective function is defined.

\subsection{Formulation of the minimization problem}

The apparent contact angle in the Cassie-Baxter state is given as follows: $\cos \theta^{*}=-1+\phi(1+\cos \theta)$. From a plot of $\theta^{*}$ versus spacing ratio $\phi$ for a constant $\theta$, it can be deduced that the apparent contact angle increases monotonously as $\phi$ is reduced. Hence, for the sake of simplicity, $\phi$ is chosen as the objective of the minimization problem, given as follows:

$$
\text { minimize } f(a, b, c)=\left(\frac{a}{a+b}\right)^{2} \text {. }
$$

Table 1 summarizes the three constraints and their physical significance.

The following values are assigned to the constants featured in these equations: acceleration due to gravity $g-$ $9.81 \mathrm{~m} / \mathrm{s}^{2}$, liquid-air interfacial surface tension $T-$ $0.072 \mathrm{~N} / \mathrm{m}$ [17], radius of the spherical water droplet $1 \mathrm{~mm}$, contact angle made by water droplet over Teflon surface $-110^{\circ}[18]$ and density of water $-1000 \mathrm{~kg} / \mathrm{m}^{3}$.

This analysis is for sharp edged pillars. However, there are limitations in fabricating sharp edged pillars, more so with photolithography and chemical etching. This may lead to slight rounding of the pillar edges. This formulation has therefore been modified to consider these aspects as discussed here.

\subsection{Modelling square arrayed surface with round edged pillars}

In this analysis, the sharp edged pillars are replaced by round edged pillars with radius of curvature $R_{c}$, see figure 7 .

Table 1. Constraint inequality and their physical significance.

\begin{tabular}{lc}
\hline Inequality & Physical significance \\
\hline$\left(\frac{1-\sin \theta}{-2 \cos \theta}\right)-\frac{c}{b}<0$ & Lower meniscus should not touch the bottom surface \\
$b+a-\left(\sqrt{\frac{-3 a T \sin ^{2} \theta^{*} \cos \theta}{\rho R g}\left(\frac{4}{\left(1-\cos \theta^{*}\right)^{2}\left(2+\cos \theta^{*}\right)}\right)^{\frac{2}{3}}+a^{2}}\right)<0$ & Drop weight balanced by surface tension \\
$\cos \theta+\left(1 / 1+\frac{4 a c}{b^{2}+2 a b}\right)<0$ & Cassie-Baxter state is more favourable than Wenzel state
\end{tabular}
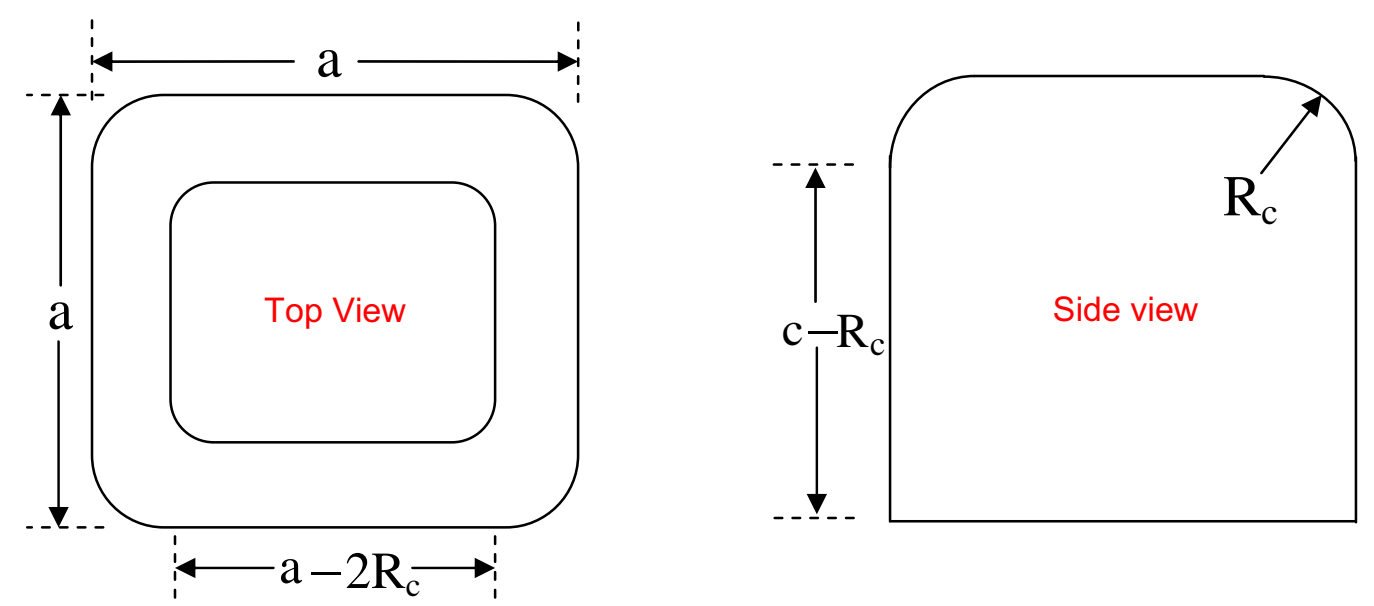

Figure 7. Schematic showing the top and side views of the round edged pillars with radius of curvature $R_{c}$. 
Table 2. Mathematical constraints and their physical significance for round edged pillars.

\begin{tabular}{lc}
\hline Inequality relation & Physical significance \\
$\frac{-b(1-\sin \theta)}{2 \cos \theta}-\left(c-R_{c}\right)<0$ & Lower meniscus should not touch the bottom surface \\
$b+a<\left[\left(\left(a-2 R_{c}\right)^{2}-\frac{3 T \cos \theta \sin ^{2} \theta^{*}\left(a-2 R_{c}\right)}{\rho g R}\right)\left\{\frac{4}{\left(1-\cos \theta^{*}\right)^{2}\left(2+\cos \theta^{*}\right)}\right\}^{\frac{2}{3}}\right]^{\frac{1}{2}}$ & Drop weight balanced by surface tension \\
$\cos \theta+1 /\left(1+\frac{4 a\left(c-R_{c}\right)}{b^{2}+2 a b}\right)<0$ & Cassie-Baxter state is more favourable than Wenzel state \\
\hline
\end{tabular}

The optimum dimensions are determined by formulating a similar minimization problem considering identical physical constraints and set of assumptions as given earlier. The objective therefore is to maximize the apparent contact angle for a single droplet resting on its surface. Table 2 presents a list of mathematical constraints and their physical significance in the case of round edged pillars.

\subsection{Round edged pillars versus sharp edged pillars}

The surface energies of a droplet placed on sharp and round edged pillared surfaces are compared. It is assumed that both the surfaces are in the Cassie-Baxter state. The energy associated with the round edged pillar surface per 'unit cell' is given by the following equation after suitably modifying Eq. (9):

$$
\begin{aligned}
E_{c 1}^{\prime}= & \gamma_{s l}\left[\left\{2\left(\frac{a}{2}-R_{c}\right)\right\}^{2}+\frac{2 \pi R_{c}}{4}\left\{2 a+2\left(a-2 R_{c}\right)\right\}\right] \\
& +\gamma_{s v}\left[(a+b)^{2}-a^{2}+4 a\left(c-R_{c}\right)\right]+\gamma\left[(a+b)^{2}-a^{2}\right] .
\end{aligned}
$$

If the droplet on round edged pillar surface has to be more stable, then $E_{c 1}^{\prime}<E_{c 1}$; substituting $E_{c 1}$ and $E^{\prime}{ }_{c 1}$ from Eqs. (9) and (11), respectively, and using Young's relation, we obtain

$$
R_{c}>\frac{\left(\frac{\pi a}{2}-\frac{\gamma_{s v}}{\gamma_{s l}}\right)}{\left(\frac{\pi-2}{2}\right)}
$$

Notice that the round edged pillar surface leads to an energetically more favourable state as compared with the sharp edged pillar surface, provided that the radius of the round corners is greater than a certain critical value. This critical value is dependent upon the width of the pillars and the ratio of the interfacial surface tension of solid-vapour to that of the solid-liquid pair.

\subsection{Additional constraints}

In the minimization problem, some additional constraints on resolution and accuracy of fabrication process have to be added to avoid trivial solutions and get optimum values of $\left(a, b, c, R_{\mathrm{c}}\right)$ that are feasible from manufacturing perspective. Additional constraints are as follows:

i. Avoiding trivial solutions: $\{a, b, c\}>0$.

ii. Practical feasibility: Very large pillar heights are infeasible from manufacturing standpoint. Hence, an upper bound has been specified, $c<c_{\text {max }}$.

iii. Manufacturing method resolution and accuracy: The fabricated pillars have to be adequately separated so that they do not overlap. This is specified as $b>10 \mu \mathrm{m}$. Also, for any fabrication method, there is a lower limit on the width of the pillars, which can be obtained with consistency and accuracy. This is specified as $a>$ $25 \mu \mathrm{m}$, which can typically be achieved. Better feature size than $25 \mu \mathrm{m}$ can indeed be achieved as has recently been demonstrated by Worgull et al [19].

\section{Results and discussion}

The constrained minimization problem is solved to minimize the spacing ratio $\phi$, which corresponds to maximum contact angle $\theta^{*}$, and the optimum dimensions $a, b, c$ are obtained (by the trust region reflective algorithm) using Matlab ${ }^{\circledR}$.

\subsection{Optimum solution}

The optimum results from the solver are presented in table 3 .

It is observed that both the sharp edge and round edge pillared surfaces can exhibit super-hydrophobicity with respective maximum contact angles $165.09^{\circ}$ and $164.37^{\circ}$. The optimum pillar heights are $c=931 \mu \mathrm{m}$ and $c=$ $1013 \mu \mathrm{m}$, respectively, for sharp and round edge pillars. The relatively large pillar height is because of the imposed constraints of lower meniscus not allowed to touch the bottom surface, and the Cassie-Baxter state being more favourable than the Wenzel state. The latter condition requires that the surface energy associated with dry cavities should be lower than wet cavities, which occurs for large pillar heights. However, the heights are large from manufacturing point of view. Hence, an upper limit on the maximum allowable pillar height $c_{\max }$ is considered in this 
Table 3. Optimum dimensions for maximum apparent contact angle for sharp and round edged pillared surfaces when there is no upper limit on $c$.

\begin{tabular}{lccccc}
\hline & $\begin{array}{c}\text { Height } \\
\text { of the } \\
\text { pillars } \\
c(\mu \mathrm{m})\end{array}$ & $\begin{array}{c}\text { Width } \\
\text { of the } \\
\text { pillars } \\
a(\mu \mathrm{m})\end{array}$ & $\begin{array}{c}\text { Spacing } \\
\text { between } \\
\text { pillars } \\
b(\mu \mathrm{m})\end{array}$ & $\begin{array}{c}\text { Maximum } \\
\text { apparent } \\
\text { contact } \\
\text { angle } \theta^{*} \\
(\mathrm{deg})\end{array}$ & $\begin{array}{c}\text { Radius } \\
\text { of round } \\
\text { corners } \\
R_{c}(\mu \mathrm{m})\end{array}$ \\
$\begin{array}{l}\text { Geometry } \\
\text { type }\end{array}$ & 931 & 25 & 85 & 165.09 & NA \\
\hline $\begin{array}{c}\text { Sharp } \\
\text { edges } \\
\begin{array}{c}\text { Round } \\
\text { edges }\end{array}\end{array}$ & 1013 & 25 & 80 & 164.37 & 2.5 \\
\hline
\end{tabular}

analysis. In the following sections, the effect of $c_{\text {max }}$ on the maximum attainable contact angle and the corresponding pillar dimensions is analysed.

\subsection{Effect of decreasing maximum allowable pillar height $c_{\max }$}

The effect of putting an upper limit on the maximum permissible height of pillar $c$, in the optimization problem, on the maximum apparent contact angle $\theta^{*}$ and the optimum pillar height $c$ has been presented, respectively, in figures 8 and 9.

3.2a Effect on maximum contact angle: The plot in figure 8 shows that the apparent contact angle decreases as the maximum allowable pillar height $c_{\max }$ is reduced from its maximum value of $1 \mathrm{~mm}$, for both the sharp edge and round edge pillared surfaces. Notice that the contact angle is almost independent of the pillar height, for large values of pillar height $(>0.24 \mathrm{~mm})$. The reason for this can be gathered by examining Eq. (9), where $c$ is in the denominator. Therefore, the effect of pillar height becomes insignificant at larger values of pillar height. Also, the

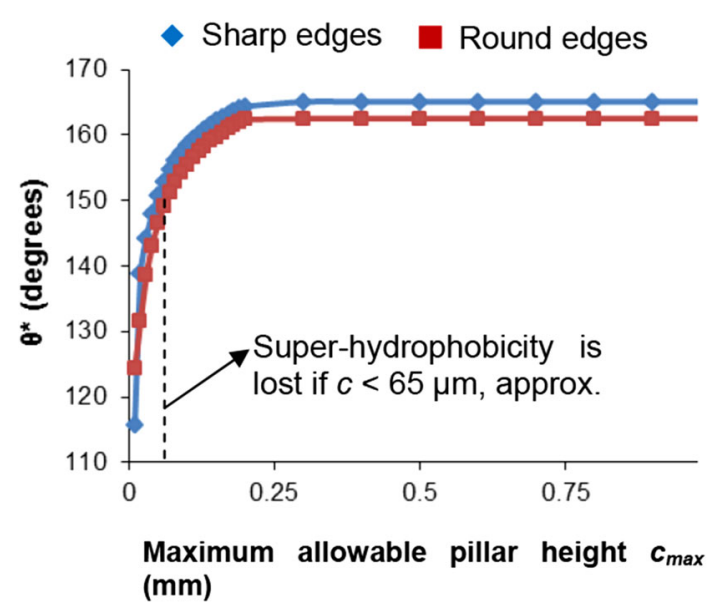

Figure 8. Apparent contact angle as a function of maximum allowable pillar height $c_{\max }$. apparent contact angles for the round edge pillars are marginally smaller than that of the sharp edge pillar.

In both the cases, the apparent contact angle remains in the super-hydrophobic region (i.e., $\theta^{*}>150^{\circ}$ ) as long as the maximum allowable pillar height is approximately greater than $65 \mu \mathrm{m}$. Hence, $c=65 \mu \mathrm{m}$ is approximately the minimum height of the pillars required to get a super-hydrophobic surface for the conditions of the surface and fluid considered here $\left(\theta=110^{\circ}\right.$, water-air surface tension $=$ $0.07 \mathrm{~N} / \mathrm{m}, \rho=1000 \mathrm{~kg} / \mathrm{m}^{3}, g=9.81 \mathrm{~m} / \mathrm{s}^{2}$, droplet radius $R=1 \mathrm{~mm}$ ). As these properties change, the minimum pillar height required to attain a super-hydrophobic surface will also change. In particular, as the contact angle between the flat solid substrate and liquid droplet $\theta$ decreases, the minimum pillar height $c$ required to achieve minimum super-hydrophobicity increases.

For the current case of $\theta=110^{\circ}$, the optimum solution set for minimum super-hydrophobicity is $\{a, b, c\}=\{25,32$, $50\} \mu \mathrm{m}$ for the sharp edge pillars, and $\left\{a, b, c, R_{\mathrm{c}}\right\}=\{25$, $37,65,2.5\} \mu \mathrm{m}$ for the round edge pillars.

3.2b Effect on optimum pillar height: The effect of decreasing the maximum allowable pillar height $c_{\max }$ on the optimum pillar height is shown in figure 9 and is discussed here.

(a) As the maximum allowable pillar height $c_{\max }$ decreases from $1 \mathrm{~mm}$, the optimum pillar height $c$ first decreases and then increases for both the sharp edge and round edged pillars (see figure 9). Figure 8 shows that in the height range $0.8 \mathrm{~mm}<c_{\max }<1 \mathrm{~mm}$, the lower height of round edge pillars gives contact angles that are marginally smaller than those for the sharp edge pillars. Hence, the optimum pillar height required, $c$, is lower for the round edge pillars than the sharp edge pillars. We know that during fabrication usually round edged pillars are generated. Our results suggest that the round edged pillars reduce the optimum pillar height required without significant

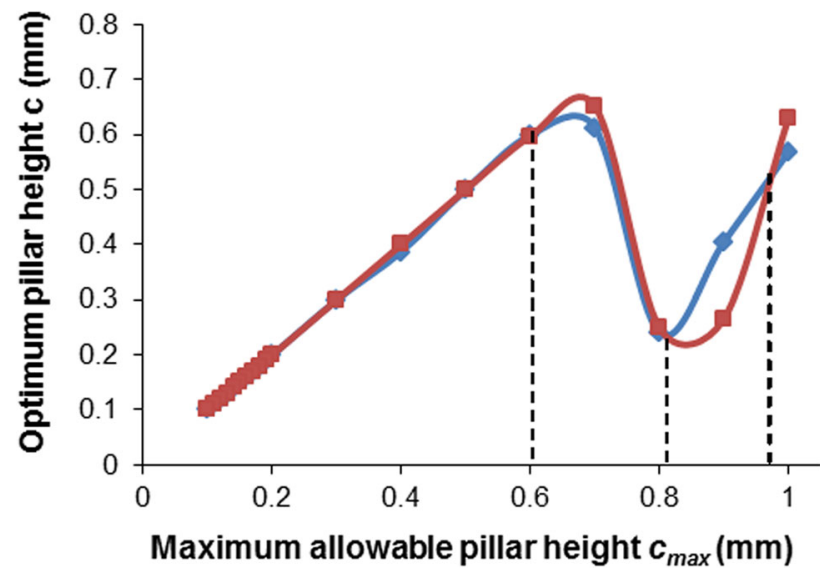

Figure 9. Variation of optimum pillar height with maximum allowable pillar height in the constraint inequality relation for sharp and round edged pillars. 
compromise on contact angle. Thus, rounding of pillars is beneficial in this height range.

(b) On the other hand, in the range: $0.6 \mathrm{~mm}<c_{\max }<0.8$ $\mathrm{mm}$ (figure 9), the optimum pillar height for the round edged pillars is more than the sharp edge pillars. Considering that there is a concurrent reduction in the contact angle (figure 8), the rounding of pillars in this range could be counter-productive.

(c) Finally, for $c_{\max }<0.6 \mathrm{~mm}$, the optimum heights of sharp and round edged pillars are almost identical. The reduction in the contact angle is similar to that of the aforementioned two cases. Therefore, round edged pillars are acceptable in place of sharp edge pillars in this range.

\subsection{Effect of changing size of droplets}

To analyse the effect of droplet size variation, optimization has been carried out by choosing the maximum pillar height, $c_{\max }=65 \mu \mathrm{m}$. Our simulation results show that when the droplet size is between 1 and $20 \mathrm{~mm}$, there is no significant change in the contact angle (see figure 10). However, when the droplet size is greater than $20 \mathrm{~mm}$, the contact angle decreases; thereby, the surface loses superhydrophobicity.

The capillary length, $k^{-1}=\left(\frac{T}{\rho g}\right)^{\frac{1}{2}}$, defined as the maximum droplet size for which gravitational forces are negligible compared with surface tension forces, below which the droplet remains spherical, has a maximum value of 2-3 mm [5]. The simulation results in figure 10 show that for the chosen geometry of pillars, a spherical droplet shows super-hydrophobic contact angle till it reaches a size of about $25 \mathrm{~mm}$, which is much larger than the size of real spherical droplets evaluated using capillary length (2-3 mm). Hence, a super-hydrophobic surface should

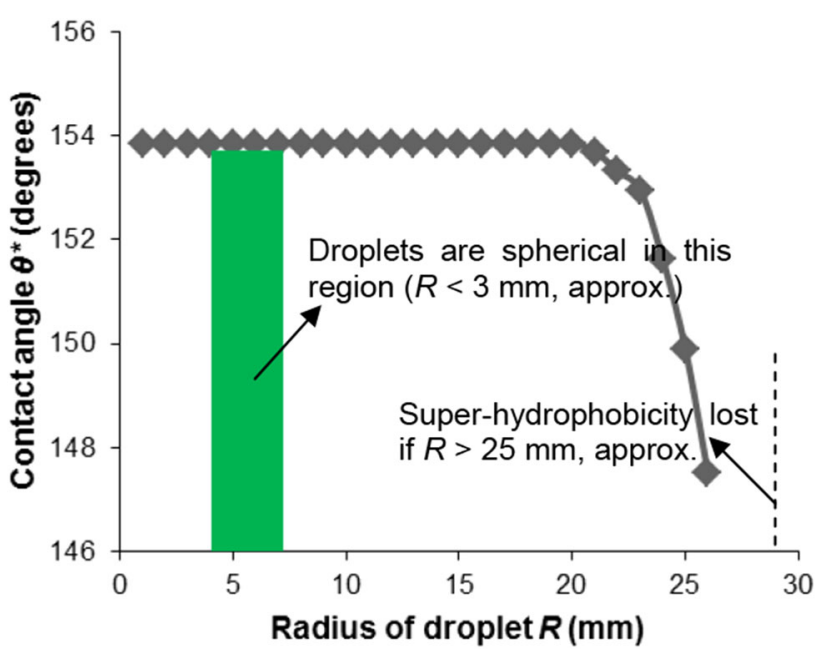

Figure 10. Apparent contact angle decreases as radius of the droplet becomes on the order of centimetres. remain super-hydrophobic for all sufficiently large spherical droplets.

\subsection{Effect of changing radius of curvature of round edge pillars}

In the earlier sections, the radius of curvature of round edges on the pillars $R_{c}$ has been considered to be $10 \%$ of the pillar width $a$. Here, the optimum dimensions are obtained as a function of $R_{c}$ and are expressed as a percentage of $a$. In the further analysis, the constrain on the upper limit of pillar height $c$ is removed, and the contact angle between the droplet and the flat surface $\theta$ is fixed at $100^{\circ}$. It is observed that a change in the radius of curvature $R_{c}$, up to $30 \%$ of $a$, reduces the contact angle from $161^{\circ}$ to $156^{\circ}$ (see figure 11). However, the optimum pillar height is found to be minimum for $R_{c}$ equal to $\sim 10 \%$ of $a$ (see figure 12 ). Hence, although rounding the pillars does not change the contact angle substantially, the pillar height required for equally large contact angle reduces, thus simplifying manufacturing.

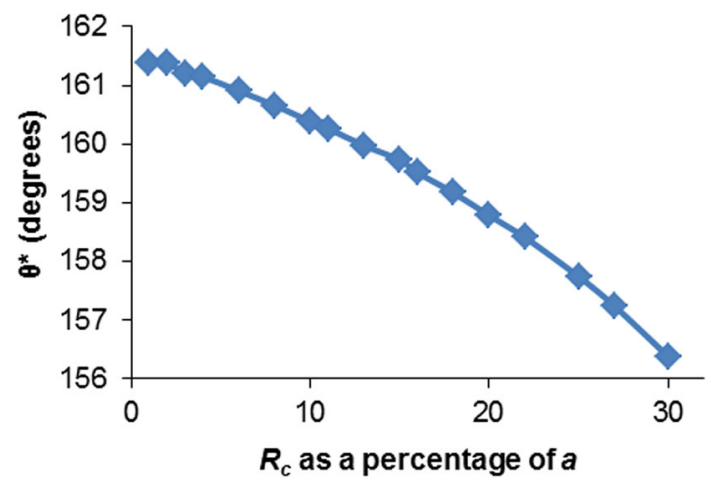

Figure 11. Optimum contact angle decreases monotonously as the curvature of pillars is increased though at a small rate.

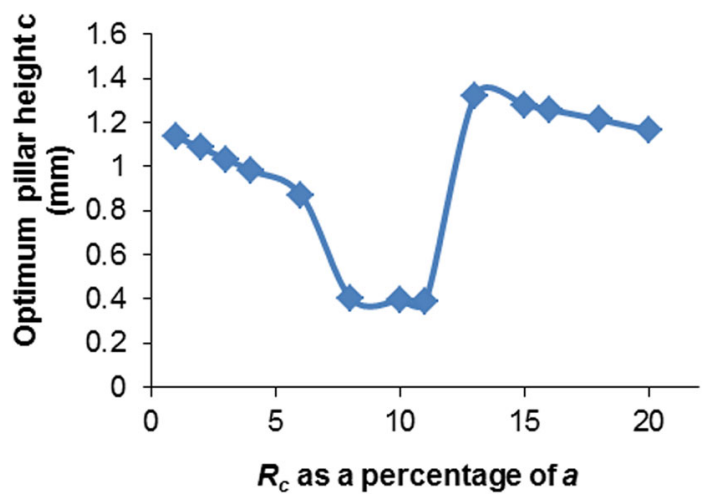

Figure 12. Optimum pillar height $c$ versus radius of curvature of pillar $R_{c}$ as a percentage of pillar width $a$. 


\section{Single-droplet simulations}

Simulations of a single droplet falling over textured surface under gravity were carried out using a commercial software $\left(\right.$ COMSOL Multiphysics $\left.{ }^{\circledR}\right)$. The purpose of these simulations is to estimate the time required for the air to escape from surface cavities, eventually leading to loss of superhydrophobicity.

\subsection{Geometry definition}

A two-dimensional axi-symmetric model is selected for the simulations. The flow is considered two-phase and laminar, and the level set function is invoked for the calculations. Table 4 and figure 13 present the constants and geometry used in the simulation, respectively. A single droplet deposited over the texture in the form of circular rings has

Table 4. Constants used in the simulations.

\begin{tabular}{lcc}
\hline Description & Expression & Units \\
\hline Density of water & 1000 & $\mathrm{~kg} / \mathrm{m}^{3}$ \\
Viscosity of water & $10^{-3}$ & $\mathrm{~kg} /(\mathrm{m} \mathrm{s})$ \\
Density of air & 1 & $\mathrm{~kg} / \mathrm{m}^{3}$ \\
Viscosity of air & $10^{-5}$ & $\mathrm{~kg} /(\mathrm{m} \mathrm{s})$ \\
Acceleration due to gravity & -9.81 & $\mathrm{~m} / \mathrm{s}^{2}$ \\
Water-air surface tension & 0.07 & $\mathrm{~N} / \mathrm{m}$ \\
\hline
\end{tabular}

been considered in the simulations. At time $t=0 \mathrm{~s}$, the droplet is completely surrounded by air and is dropped freely from a small distance of $\sim 1.5 \mathrm{~mm}$ above the solid surface.

\subsection{Boundary conditions}

At the left boundary, symmetry condition is specified. A slip boundary condition is applied on all the walls and the ceiling (see figure 13). At the bottom surface, which has textures, a wetted wall condition is applied with contact angle of $\theta=135^{\circ}$ and $\theta=90^{\circ}$ in first and second sets of simulations, respectively. A pressure of $0 \mathrm{~Pa}$ is specified at the top right corner.

\subsection{Simulation results and discussion}

The simulation results for $\theta=135^{\circ}$ are shown in figure $14 \mathrm{a}-\mathrm{e}$. The dimensions of the surface textures are indicated below each figure. The data are taken at arbitrary time instances as indicated on top of the figures. Note that the shape of the droplet may change with time. In these figures, red corresponds to $100 \%$ air, while blue corresponds to $100 \%$ water.

Figure $14 \mathrm{a}-\mathrm{c}$ shows that the liquid penetrates into the cavities, though to a lesser extent in figure 14c. It is understood that the liquid penetrates the cavities if the

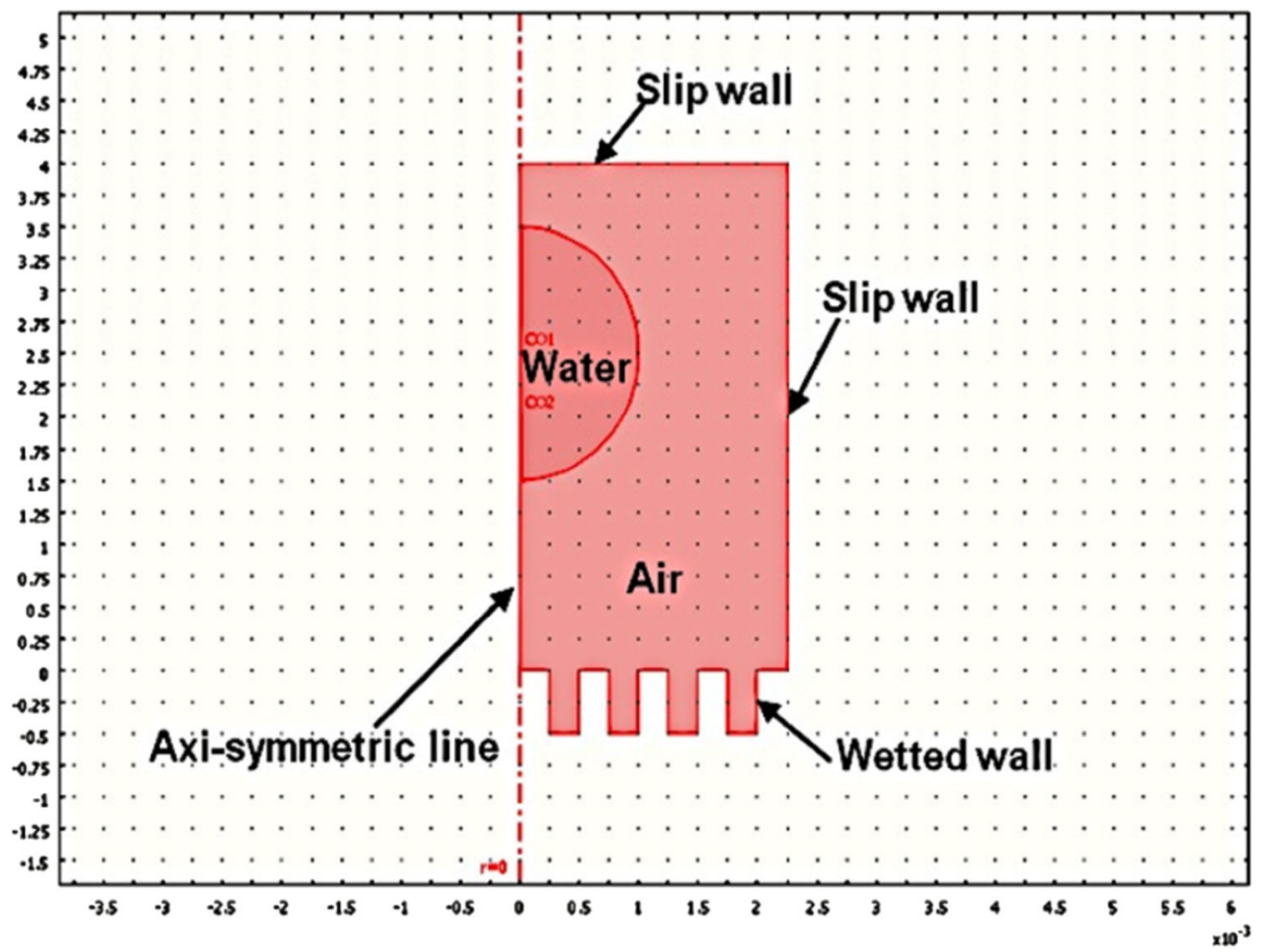

Figure 13. Geometry modelled (abscissa and ordinate are in metres). 


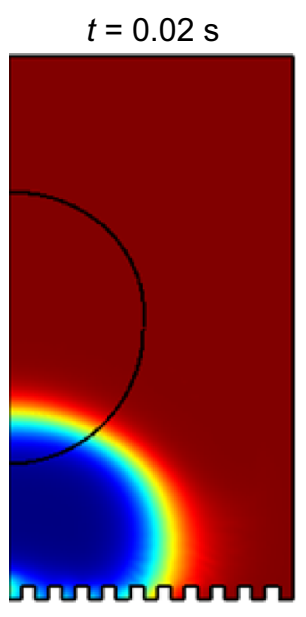

(a)

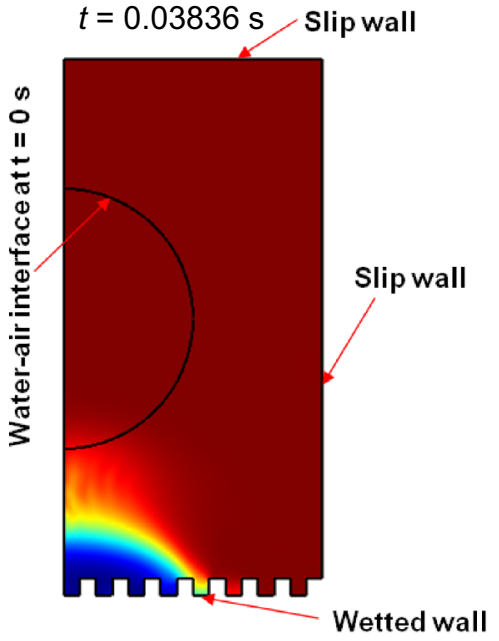

(b)

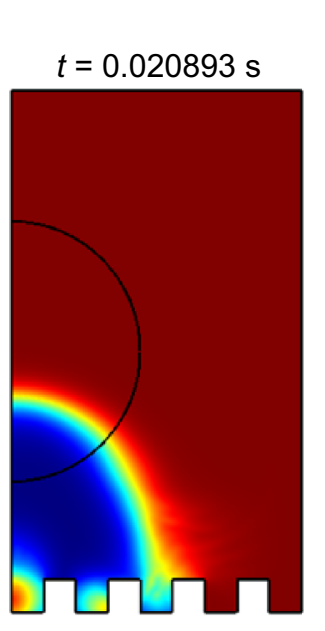

(c)
$100 \%$ Air

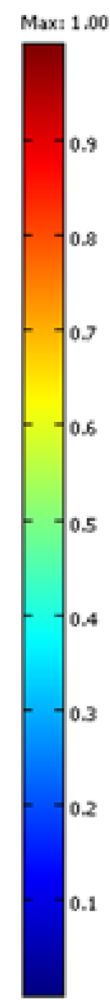

$100 \%$ Water

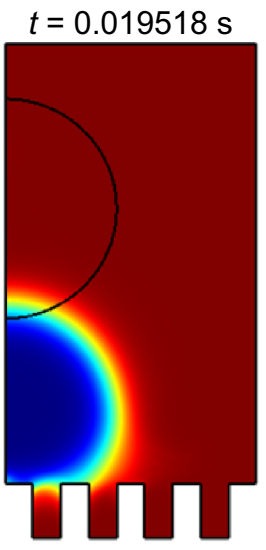

(d)

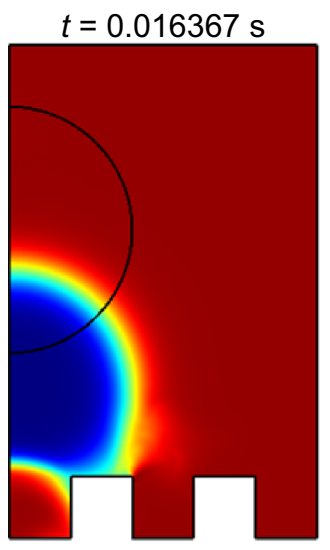

(e)

Figure 14. (a)-(e) Simulation results of a single droplet dropped over textured surfaces having intrinsic contact angle of $\theta=135^{\circ}$ with water. Notice that five different set of dimensions of the textured surfaces are being considered. (a) $a=100, b=100, c=100 \mu \mathrm{m},(\mathbf{b}) a=$ $125, b=125, c=125 \mu \mathrm{m},(\mathbf{c}) a=250, b=250, c=250 \mu \mathrm{m},(\mathbf{d}) a=250, b=250, c=500 \mu \mathrm{m}$ and (e) $a=500, b=500, c=500 \mu \mathrm{m}$.

height of the pillars is small, irrespective of the pillar spacing in the range tested. Figure $14 \mathrm{c}$ and $\mathrm{d}$ shows that the liquid does not penetrate the cavities when the pillar height is increased from 250 to $500 \mu \mathrm{m}$ for the same pillar width and spacing of $250 \mu \mathrm{m}$. Also, from figure $14 \mathrm{~d}$ and e, it is seen that the amount of liquid penetrating between the pillars is larger when the spacing between the pillars is increased from 250 to $500 \mu \mathrm{m}$, for the same pillar height of $500 \mu \mathrm{m}$.

Similar results were observed for the case of $\theta=90^{\circ}$. In this case, shapes of the droplets changed due to difference in their wetting characteristics. Therefore, it can be interpreted that the simulation results are indeed influenced by the solid-liquid interfacial surface tension.

The converged solution for $a=250 \mu \mathrm{m}, b=250 \mu \mathrm{m}, c=$ $500 \mu \mathrm{m}$ and $\theta=135^{\circ}$ is shown in figure $14 \mathrm{~d}$. Figure 15

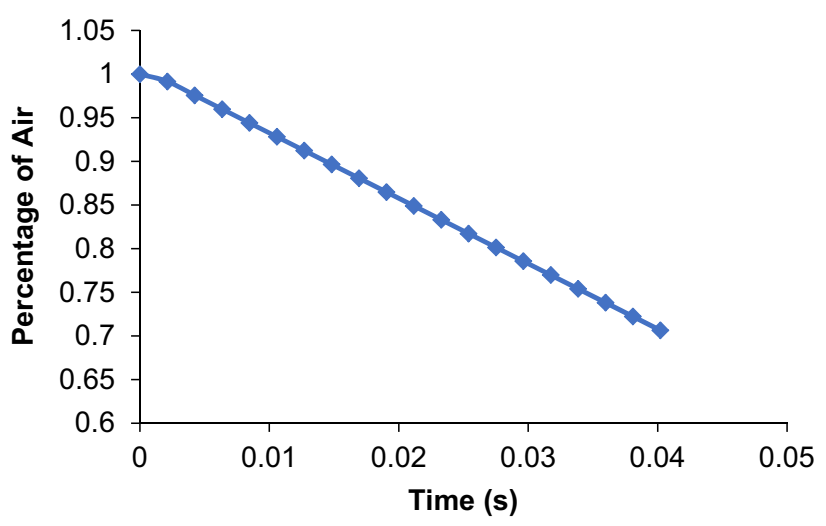

Figure 15. Percentage of air remaining in a cavity as a function of time. 


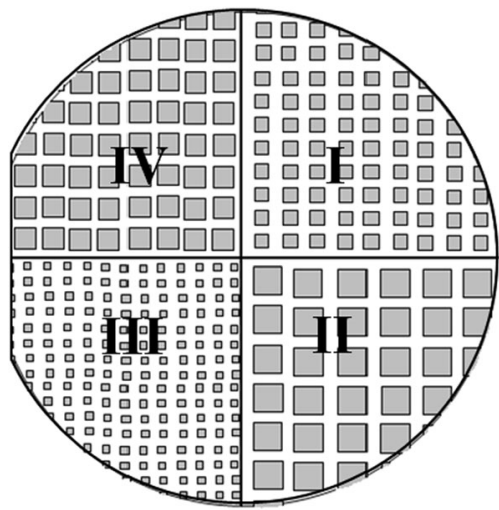

Figure 16. Photographic film (2.5 in) has square arrays of four different dimensions in the four quadrants to obtain four types of surfaces on a single silicon wafer.

shows the percentage of air as a function of time in the first cavity (from left). It is observed that the percentage of air trapped in the cavity decreases continuously. Figure $14 \mathrm{~b}$ suggests that the cavity closer to the axis gets flooded before those away from the axis. In due course, the trapped air escapes out completely, causing breakdown of superhydrophobicity. This is in conformity with the observations of Govardhan et al [20], where super-hydrophobic contact angles were seen to decrease with time. Xiu et al [21] reported that in their experiments the contact angle decreased from $158^{\circ}$ to $140^{\circ}$ over a period of 44 days, while the surface lost super-hydrophobicity in the span of just 3-4 days. Further, Wang et al [22] found out that for a droplet with the impact velocity of $0.56 \mathrm{~m} / \mathrm{s}$ on the surface, the amount of liquid penetration between pillars kept on increasing with time until the Wenzel state was reached.

\section{Fabrication of pillared structures by photolithography and model validation}

To validate the simulation results, micro-textures were fabricated on Si wafers using photolithography. In this case, micro-textures were fabricated on a SU-8 photoresist on a $\mathrm{Si}$ wafer (2.5 in) as the substrate. A mask of diameter 2.5 in
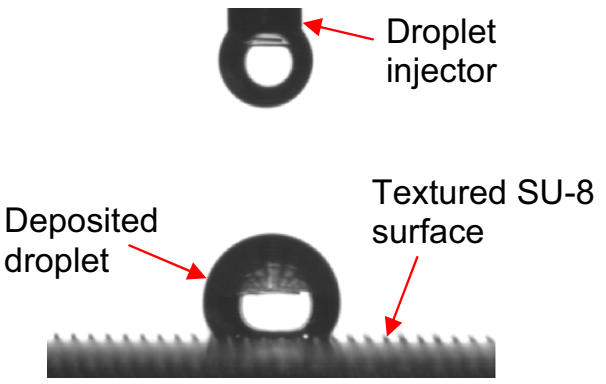

Figure 17. A contact angle of $120.5^{\circ}$ is seen on $\mathrm{Si}-\mathrm{Su}-8$ surface in quadrant III.

that has different geometries in every quadrant was used to make pillars of different geometry on the wafer surface. As SU-8 is a negative photoresist, a negative mask is required to obtain pillars on the wafer surface. The black locations on the pillars correspond to position of pillars that get formed on the wafer surface. The four quadrants (see figure 16) of arrayed pillars had different widths $a$ and spacings $b$ so as to achieve four different contact angles.

The dimensions $a$ and $b$ in each of the four quadrants (IIV in figure 16) and the contact angles formed by a sessile water droplet were measured using an optical microscope and contact angle measuring machine, respectively. The height of the pillar was interpreted by drawing the surface profile using an XP-2 Ambios Technology stylus profilometer capable of measuring heights in the range of $15 \mathrm{~nm}-200 \mu \mathrm{m}$. The pillar dimensions in the four quadrants are shown in table 5 . The contact angle was measured using a goniometer (Digidrop, GBX). The contact angle made by water on flat SU-8 surface is $78 \pm 2^{\circ}$ [23], which is in agreement with the accepted contact angle of $80^{\circ}$. The largest contact angle of $120.5^{\circ}$ (see figure 17) was observed in quadrant III.

\subsection{Comparison between experimental contact angles and predictions by model}

The Cassie-Baxter relation, which considers only the width and spacing between the pillars, predicts contact angles that

Table 5. Measured dimensions and contact angles and their comparison with theoretical Cassie and Wenzel angles, and with the angles predicted by the model in the four quadrants of the SU-8 coated Si wafer.

\begin{tabular}{|c|c|c|c|c|c|c|c|c|}
\hline \multirow[b]{2}{*}{ Quadrant } & \multicolumn{3}{|c|}{$\begin{array}{l}\text { Obtained dimensions } \\
\qquad(\mu \mathrm{m})\end{array}$} & \multirow[b]{2}{*}{ Spacing ratio $\varphi$} & \multirow[b]{2}{*}{ Measured angle (deg) } & \multicolumn{2}{|c|}{$\begin{array}{c}\text { Theoretically predicted } \\
\text { angles using existing models } \\
\text { (deg) }\end{array}$} & \multirow{2}{*}{$\begin{array}{l}\text { Model prediction using } \\
\text { the proposed model (deg }\end{array}$} \\
\hline & $a$ & $b$ & $c$ & & & Cassie & Wenzel & \\
\hline I & 125.7 & 95.5 & 46.2 & 0.323 & 74.3 & 128.4 & 75 & 130 \\
\hline II & 93.2 & 71.3 & 3 & 0.321 & 108.6 & 128.6 & 80 & 100 \\
\hline III & 109.4 & 51.9 & 75 & 0.460 & 120.5 & 117.4 & 67 & 138 \\
\hline IV & 68.2 & 76 & 17.5 & 0.224 & 105.3 & 137.5 & 78 & 100 \\
\hline
\end{tabular}




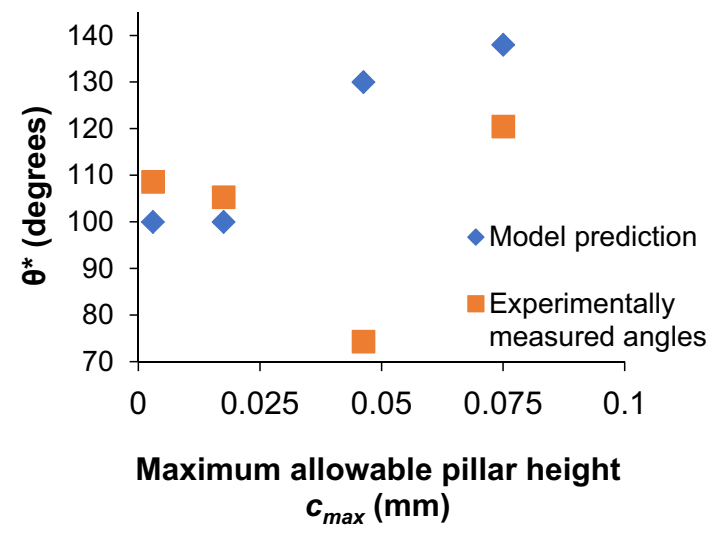

Figure 18. Comparison of experimentally measured contact angle against predictions from model in quadrants $\mathrm{I}-\mathrm{IV}$ of $\mathrm{Si}$ wafer.

are much larger than those actually obtained. On the other hand, the Wenzel model, which considers all three dimensions, predicts contact angles that are smaller than those obtained experimentally. Our model, which takes into account the pillar height along with the pillar width and spacing between the pillars, predicts the maximum attainable contact angle for a given pillar height. Assuming that the droplet is in the ideal Cassie-Baxter state, an analysis is presented for $\theta=80^{\circ}$ in figure 18 for comparison to experimental results (contact angle made by water droplet over flat SU-8 is $80^{\circ}$ as already noted).

In quadrant $\mathrm{I}$, the measured contact angle is much smaller than the predicted maximum contact angle, but is close to the angle predicted by the Wenzel model (table 5). It is possible that the highly non-uniform surface profile causes the effective pillar height to be much smaller than $46.2 \mu \mathrm{m}$, which can lead the droplet to attain the Wenzel state.

In quadrants II and IV, the measured contact angle lies between the predictions of Cassie and Wenzel models, indicating that the droplet is present in an intermediate state. The measured contact angles are slightly larger than the maximum angle predicted by the present model. The measured contact angles are closer to the present model than the two standard models. It can be interpreted that the non-uniformity of pillars introduced during fabrication causes the effective pillar height to be larger than 3 and $17.5 \mu \mathrm{m}$ in quadrants II and IV, respectively.

In quadrant III, the measured contact angle is close to the prediction of the Cassie model. The measured contact angle is smaller than the predicted maximum contact angle obtained using the present model. This can possibly be attributed to the obtained dimensions $a$ and $b$ being different from the optimum dimensions.

It is observed that the uniformity in pillar heights is relatively better in quadrant III; further, the pillars are of significantly larger heights as compared with the pillars obtained in the other three quadrants. This is possibly the reason for the largest contact angle (of $120^{\circ}$ ) attained in quadrant III as compared with the other three quadrants. In the other quadrants, non-conformity between the measured contact angles and those predicted by the model can be possibly attributed to non-uniformity in the pillar dimensions, including height and spacing. Note that notwithstanding these limitations, the predicted contact angles compare well to the measured ones. Therefore, better agreement between the predicted and measured values of contact angles can be achieved if the dimensions of the pillars fabricated are fairly uniform. On the model front, we have taken into account one of the uncertainties of the fabrication, that is rounding of the pillar edges; however, the non-uniformities in the spacing and other dimensions of the pillars need to be incorporated in the model.

\section{Concluding remarks}

- The paper presents an analytical model for predicting the contact angle of droplet on textured surfaces with straight and round edge pillars. The proposed model employs an optimization procedure to determine the absolute values of pillar dimensions. This represents an improvement over the existing models, which predict the relative dimensions only.

- The optimal dimensions predicted by the analytical model were used in the numerical simulations to determine the duration for which air remains trapped inside the surface cavities and renders super-hydrophobic property to the surface.

- The textured surfaces were also fabricated for the optimal set of dimensions to validate the proposed model. The measured contact angle was found to be in agreement to the model predictions when the fabricated pillars have fairly uniform dimensions.

- The analytical model based on Cassie-Baxter state predicts that surface textures with either sharp or round edged pillars of appropriate dimensions can exhibit super-hydrophobicity. The model demonstrated that the round edge pillars, arising out of fabrication imperfections, reduce the height of the pillars without significant compromise on the contact angle.

- The fabricated surface exhibits super-hydrophobicity for a large range of spherical droplets $(R<\sim 25 \mathrm{~mm})$. Single-droplet simulations confirm the earlier observations that the liquid penetrates the cavities if the height or spacing between the pillars is small.

- The work presented in this paper can potentially be extended to take into consideration other imperfections that creep in during fabrication of textured surfaces, as well as complexities in the contact and flow characteristics arising due to motion of droplets and/or bulk fluid over textured surfaces. 


\section{Acknowledgements}

Authors wish to express their gratitude to ISRO Cell, IIT Bombay, for financial support to undertake this work.

\section{References}

[1] Feng L, Li S, Li Y, Li H, Zhang L, Zhai J, Song Y, Liu B, Jiang L and Zhu D 2002 Super-hydrophobic surfaces: from natural to artificial. Adv. Mater. 24(14): 1857-1860

[2] Liu T, Chen S, Cheng S, Tian J, Chang X and Yin Y 2007 Corrosion behavior of super-hydrophobic surface on copper in seawater. Electrochim. Acta 52: 8003-8007

[3] Kim M, Kim K, Lee N Y, Shin K and Kim Y S 2007 A simple fabrication route to a highly transparent super-hydrophobic surface with a poly(dimethylsiloxane) coated flexible mold. Chem. Commun. 22: 2237-2239

[4] Huang X J, Lee J H, Lee J W, Yoon J B and Choi Y K 2008 A one-step route to a perfectly ordered wafer-scale microbowl array for size-dependent superhydrophobicity. Small 4(2): 211-216

[5] Quéré D 2005 Non-sticking drops. Rep. Prog. Phys. 68(11): 2495-2532

[6] Zhu L, Feng Y, Ye X and Zhou Z 2006 Tuning wettability and getting superhydrophobic surface by controlling surface roughness with well-designed microstructures. Sens. Actuators A 130-131: 595-600

[7] Van der Wal P and Steiner U 2007 Super-hydrophobic surfaces made from Teflon. Soft Matter 3: 426-429

[8] Tang M, Hong M H and Choo Y S 2008 Hydrophobic surface fabrication by laser micropatterning. In: IEEE PhotonicsGlobal@Singapore, National University of Singapore, 8-11 Dec 2008

[9] Neinhuis C and Walzel P 2005 Wetting and self-cleaning properties of artificial superhydrophobic surfaces. Langmuir 21: 956-961

[10] Li J, Wang L and Jiang W 2010 Super-hydrophobic surface of bulk carbon nanotubes compacted by spark plasma sintering followed by modification with polytetrofluorethylene. Carbon 48: 2644-2673

[11] Zhu L, Xiu Y, Xu J, Tamirisa P A, Hess D W and Wong C P 2005 Superhydrophobicity on two-tier rough surfaces fabricated by controlled growth of aligned carbon nanotube arrays coated with fluorocarbon. Langmuir 21: 11208-11212

[12] Yoon H, Park J H and Kim G H 2010 A superhydrophobic surface fabricated by an electrostatic process. Macromol. Rapid Commun. 31: 1022-1336

[13] Klein R J, Biesheuvel M, Yu B C, Meinhart C D and Lange F F 2003 Producing super-hydrophobic surfaces with nanosilica spheres. Zeitschrift für Metallkunde 94(4): 377-380

[14] Schrauth A J, Saka N and Suh N P 2004 Development of nano-structured hemocompatible surfaces. In: Proceedings of the 2nd International Symposium on Nano-Manufacturing, Massachusetts Institute of Technology, Boston, USA

[15] Sbragaglia M, Peters A M, Pirat C, Borkent B M, Lammertink R G H, Wessling M and Lohse D 2008 Spontaneous breakdown of superhydophobicity. Phys. Fluids 81: 66002-66006

[16] Koishi T, Yasuoka K, Fujikawa S, Ebisuzaki T and Zeng X C 2009 Coexistence and transition between Cassie and Wenzel state on pillared hydrophobic surface. Proc. Natl. Acad. Sci. 106(21): 8435-8440

[17] http://en.wikipedia.org/wiki/Surface_tension_values

[18] www.uweb.engr.washington.edu/.../ASHsurfscontact\% 20angles05.pdf

[19] Worgull M, Heckele M, Mappes T, Matthis B, Tosello G, Metz T, Gavillet J, Koltay P and Hansen H N 2009 Sub- $\mu$ m structured lotus surfaces manufacturing. Microsyst. Technol. 15: $1327-1333$

[20] Govardhan R N, Srinivas G S, Asthana A and Bobji M S 2009 Time dependence of effective slip on textured hydrophobic surfaces. Phys. Fluids 21(5): 052001

[21] Xiu Y, Zhu L, Hess D and Wong C P 2006 Superhydrophobicity and UV stability of polydimethylsiloxane/ polytetrafluoroethylene (PDMS/PTFE) coatings. In: Proceedings of the 11th IEEE CPMT International Symposium and Exhibition on Advanced Packaging Materials: Processes, Properties and Interfaces, Atlanta, GA, United States, March 15-17, pp. 98-103

[22] Wang Z, Lopez C, Hirsa A and Koratkar N 2007 Impact dynamics and rebound of water droplets on superhydrophobic carbon nanotube arrays. Appl. Phys. Lett. 91: 023105

[23] Gomez G B, Glidle A, Flendrig L M and Cooper J M 2009 Creation of super-hydrophobic siloxane-modified SU-8 microstructures. Microelectron. Eng. 86: 1325-1328 